\title{
Lattice QCD on new chips: a community summary
}

\author{
Antonio Rago ${ }^{1,2, \star}$ \\ ${ }^{1}$ Centre for Mathematical Sciences, Plymouth University, Plymouth, PL4 8AA, United Kingdom \\ ${ }^{2}$ CERN, Theoretical Physics Department, Geneva, Switzerland
}

\begin{abstract}
I review the most recent evolutions of the QCD codes on new architectures, with a focus on the performances obtained by the different coding strategies as presented during the Lattice2017 conference.
\end{abstract}

\section{Introduction}

In 1965 Gordon Moore formulated his famed law: companies should be capable of doubling the number of transistors on a given area of chip every two years [1]. The law has roughly held true up to now, with the caveat that the original formulation has been enlarged to include other aspects than raw single-CPU frequency scaling (see figure 1). Nowadays Moore's law is attained by the increase in the number of cores, larger vectorization units, and more performing memory structure and hierarchy. Unfortunately the interconnection speed has not kept up with the pace of progress of the other components. You may also find various claims that we are getting close to the end of the silicon era, with evolutions such as tunneling transistors and spintronic. The impending doom has been announced at least ten years ago and Moore's law is still holding, silicon-based.

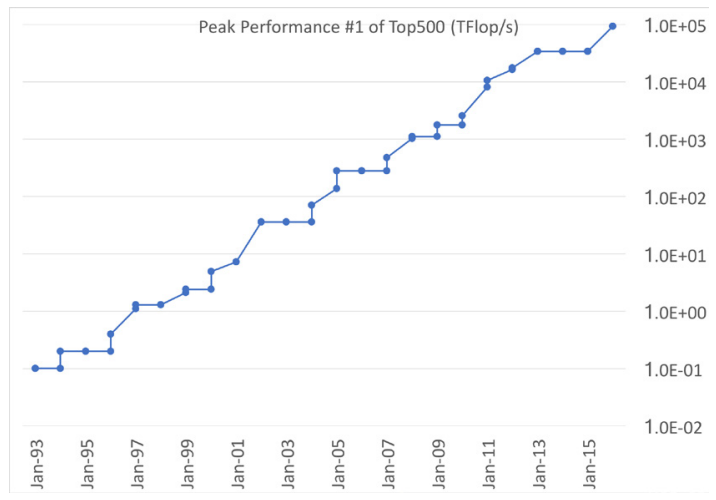

Figure 1. Peak performance of the top supercomputer of the world over the years (data from TOP500 [2]).

\footnotetext{
^Speaker, e-mail: antonio.rago@plymouth.ac.uk
} 
This hardware complexity and variety reflects into challenges for the programmer who intends to write optimised code. While in the past only scalar tuning needed to be considered to reach optimal performance, nowadays the roadmap for the HPC programmer is definitely more complex: to fully exploit the computational power of modern hardware, the programmer must think of coordination and communication, threading (MIMD) and vectorisation (SIMD). And on top of that careful data motion and memory and caching strategies need to be orchestrated too. Each of these aspects will be associated to hardware bounds (cache, memory, network ...) and the challenge programmers must face, to exploit full hardware capabilities, is to saturate all these bounds together [3].

\section{Hardware and QCD applications}

In the following I will present the efforts of the lattice community in developing code for the newest available hardware, as presented during the Lattice 2017 conference.

\subsection{Intel Xeon Phi}

The Xeon Phi name identifies a set of many core processors (MIC-many integrated core) developed by Intel and designed specifically for HPC purposes. One of the principal features of this chipset is the full $\mathrm{x} 86$ compatibility, i.e. the capability of running software targeted at standard $\mathrm{x} 86 \mathrm{CPU}$. Another important feature is the compatibility with standard programming API such as OpenMP. These features should facilitate code portability from other similar architectures.

The Xeon Phi was initially developed as a PCIe coprocessors, with the codename Knight Corner (KNC), while for the second generation it was developed also as standalone CPU structure with codename Knights Landing (KNL).

The specific structure of the KNL has been presented and discussed in many publications [3-5], in this work we only highlight the most relevant features for lattice simulations.

\subsubsection{KNL organisation}

The structure of the KNL is reported in figure 2.

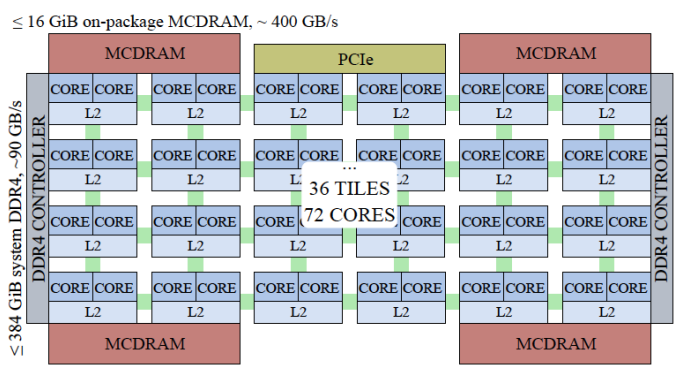

(a) Schematic diagram of the on-chip tiles arrangement on a processor.

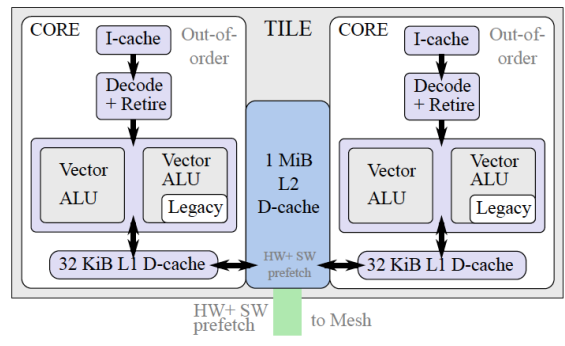

(b) Single tile structure.

Figure 2. KNL die organisation.

It consists of a set of tiles with up to 36 tiles for KNL. Each tile is equipped with 2 physical cores, for a maximum of 72 cores. Each core shares a L2 cache on the tile that is then distributed with 
interconnection mesh as per figure 2.a. Each core is optimised for 4-way hyper-threading, hence up to $4 \times 72=288$ logical processes per KNL. The KNL is equipped with an on-package high-bandwidth memory MCDRAM (16GB at 475GB/s [6]) connected by four controllers to the processing cores.

\subsubsection{KNL CPU \& VPU}

The KNL tile is organised as per figure 2.b. The structure of KNL tile is designed for an hierarchical coding paradigm with each core running multiple threads/processes (MIMD) and each thread (process) issuing vector instructions (SIMD). Each core has two Vector Processing Units (VPUs) and the out-of-order pipeline is capable of two instructions per cycle. The optimal achievable benefit due to the vectorisation is:

- Single Precision $\rightarrow 512$ bit registers $/ 32$ bits $\times 2$ VPUs $=32$ SIMD lanes.

- Double precision $\rightarrow 512$ bit registers $/ 64$ bits $\times 2$ VPUs $=16$ SIMD lanes.

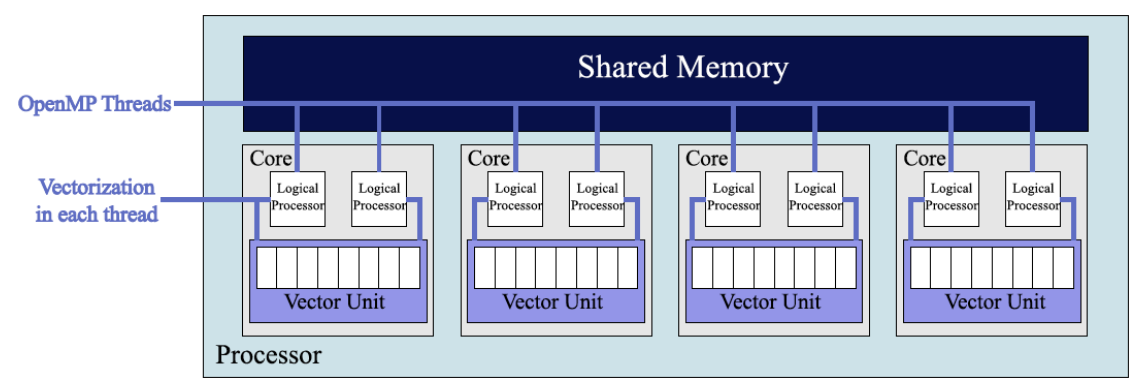

Figure 3. KNL coding hierarchy: co-existance with vectors.

\subsubsection{Key points in KNL optimisation}

While designing a code for the KNL architecture, one has to take into account the following key aspects:

- Vectorisation: Take full advantage of the 512 bit registers of the 2 VPU. Specifically noting that the legacy instructions (256 bits or less) are available only on one of the VPU, it could even be beneficial to take advantage of the compiler automatic vectorisation.

- Distributed L2 Cache with a mesh interconnect: data locality is of paramount importance. The locality can be exploited through three different clustering modes characterised by a different degree of affinity between distributed Tag Directory (TD) and cache.

The three modes are:

- All-to-All: In this mode there is no affinity between the TD and the memory (only for debug purpose).

- Quadrant/Hemisphere: In this mode the TD and the memory reside in the same quadrant/hemisphere, see figure 4.a. The partitioning of the chip into quadrants is not visible to the operating system, hence it is sufficient to maintain data access locality to take full advantage of this mode. 


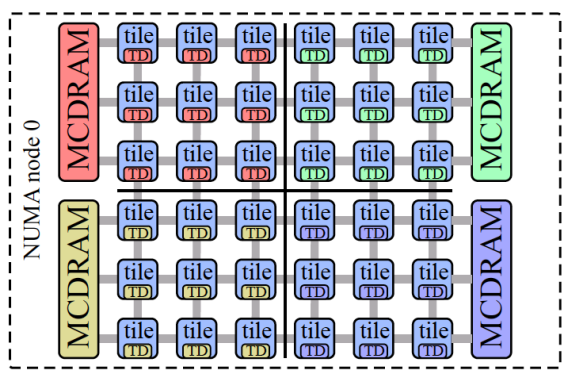

(a) Quadrant mode: Tag Directory and memory reside in the same quadrant.

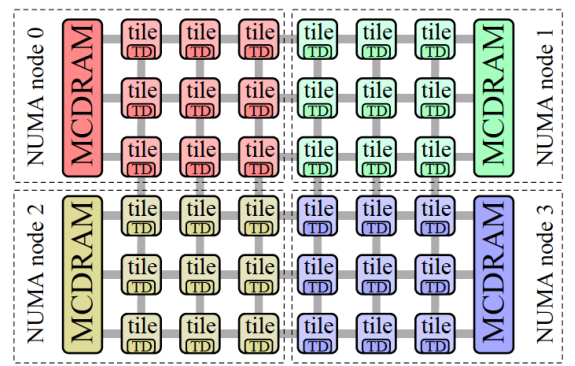

(b) SNC-4 mode: Cores appear as 4 NUMA nodes.

Figure 4. KNL clustering modes.

- SNC-4/SNC-2: In this mode the cores appear as 4 (or 2) NUMA nodes exposed to the operating system. In this case it is recommended to use a code that is NUMA aware, ensuring that each tile works mostly with its local memory, see figure 4.b.

- The MCDRAM has a bandwidth roughly 4 times bigger than the DDR4 RAM's one, making it very convenient to fit all the data in the MCDRAM and avoid the use of DDR4 for active data handling. The KNL offers 3 different memory modes (see figure 5):

- Flat Mode: In this mode the MCDRAM is treated as a NUMA node and the user controls what goes in MCRAM.

- Cache Mode: In this mode the MCDRAM is treated as Last Level Cache and it is used automatically.

- Hybrid Mode: This mode is a combination of the previous two, with the ratio between Flat and Cache that can be chosen at boot time from the BIOS.

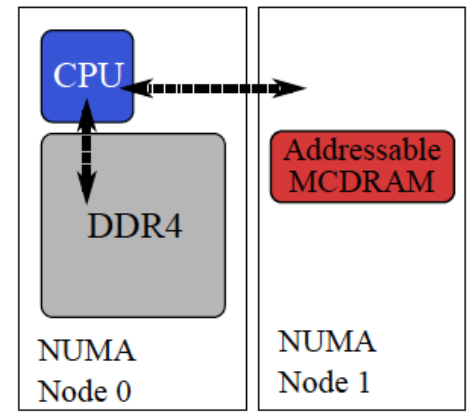

(a) Flat mode.

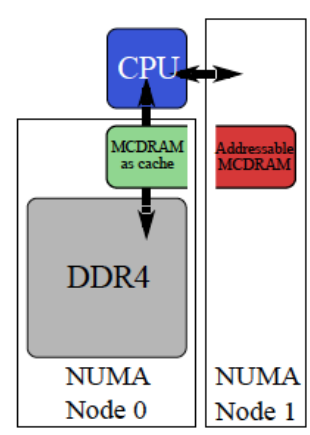

(b) Cache mode.

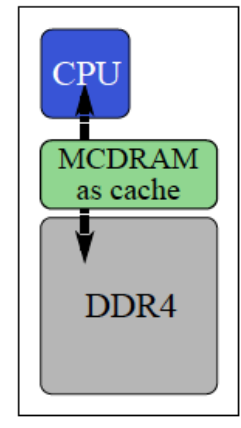

(c) Hybrid mode.

Figure 5. KNL MCDRAM memory modes.

As reference the theoretical peak performance of the KNL using single precision is of over $6 \mathrm{TFlop} / \mathrm{s}$ and over 3 TFlop/s for double precision [5]. 


\subsubsection{Contributions}

During the conference many of the talks reported on implementation and optimisation specific for the KNL architecture, with a spectrum of coding philosophy ranging from the need of portability and compatibility of the code, to implementations specialised for the KNL architecture.

Optimisation of the Brillouin operator on the KNL architecture: S. Durr presented a rather straightforward, but still performing, implementation of the Brillouin operator for the KNL architecture [7]. Brillouin is an implementation of the Dirac operator that shows better scaling properties of the dispersion relations compared to the usual Wilson Dirac operator, and it represents a suitable kernel for the overlap procedure $[8,9]$. Compared to the Wilson operator the Brillouin operator requires many more hop terms, leading to an estimate of the ratio of computational intensity of Brillouin over Wilson of 5/2. The implementation strategy for the application of the operator on multiple spinors can be schematically described as (from the innermost cycle to the outermost cycle):

1. Unroll the local color and spinor indices.

2. SIMD-ize over the index of spinor with an OMP pragma.

3. Distribute the geometry of OMP threads.

In figure 6 it is reported the flop count per second of the single precision Brillouin and Wilson operators as function of the number of threads, for number of colours $N_{C}=3$, number of spinor $N_{V}=3 N_{C}$. In the best configuration the Brillouin operator can obtain a performance of $357 / 272$

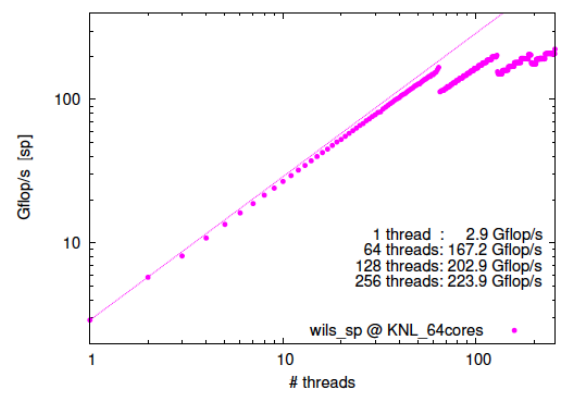

(a) Wilson operator.

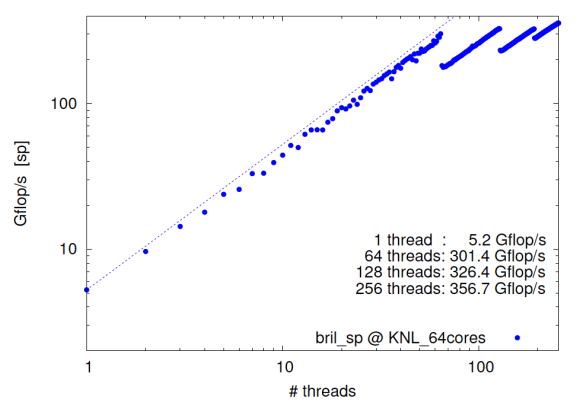

(b) Brillouin operator.

Figure 6. Scaling of the flop count per second for the matrix vector operation as function of the number of threads.

Gflop/s (peak/sustained) meaning 6.8/10.4\% of the KNL theoretical performances, while for the Wilson operator the performances are $225 / 135 \mathrm{Gflop} / \mathrm{s}$ (peak/sustained) meaning $4.3 / 5.2 \%$ of the KNL.

The takeway information is that for this kind of applications a reasonable performance can be obtained, while maintaining full portability even using simple coding strategy.

Grid software status and performance: The data parallel framework Grid is developed by the Edinburgh group: P. A. Boyle, G. Cossu, A. Yamaguchi and A. Portelli [10, 11]. One of the main features of the Grid suite is the arrangement of the data layout, that can be summarised as:

- Geometrically decompose cartesian arrays across nodes (MPI). 
- Subdivide node volume into smaller virtual nodes.

- Spread virtual nodes across SIMD lanes.

- Use OpenMP+MPI+SIMD to process conformable array operations.

- Automatically modify data layout to align data parallel operations to SIMD hardware.

The key idea is to execute exactly the same instructions on many nodes, with each node acting at the same time on multiple virtual nodes, see figure 7 . The benefit comes from the observation that conformable array operations are simple and vectorise perfectly. The performances of Grid in single

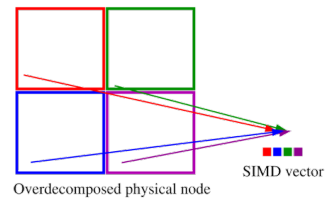

(a) Over-decompose and interleave elements from different virtual nodes in adjacent SIMD lanes.

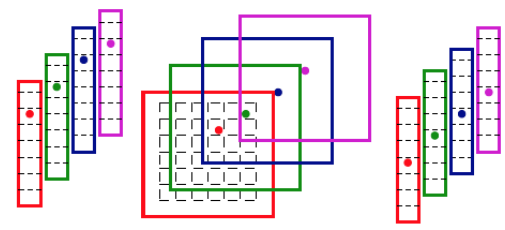

(b) SIMD accelerating matrix-vector products: Many vectors = many matrices $\mathrm{x}$ many vectors

Figure 7. Grid data layout [10].

precision on single node for multiple RHS on the Wilson operator were already presented [3, 10, 12], it is worth however to highlight the most relevant features:

- Single core instructions-per-cycle (IPC) is 1.7 (85\% of theoretical, 2 IPC).

- Multi-core L1 hit rate is $99 \%$ (perfect SFW prefetching).

- Multi-core MCDRAM bandwidth 97\% (370GB/s), with 1 thread per core fastest after writing in assembler (not intrinsics).

The group reports a very detailed breakdown of the data movement trough the system caches and compare their findings with the optimal theoretical values, see table 1.

Table 1. Grid data movement.

\begin{tabular}{ccccc}
\hline L1 read & L1 write & L2 read & MCDRAM read & MCDRAM write \\
\hline 550 & $12(12$ theo. $)$ & $98(96$ theo. $)$ & $68(12$ theo. $)$ & $12(12$ theo. $)$ \\
\hline
\end{tabular}

From the ratio of the L2 read over the MCRDAM read they evaluate the reuse factor $(98 / 68=$ $1.44)$ that is well below the ideal case of infinite L2 capacity $(96 / 12=8)$. The data seems to suggest this inefficiency is due to a non optimal scaling of the interconnection mesh of the L2 cache on the KNL. Finally for the single node single precision assembler Domainwall Dirac operator and with the described data layout Grid achieves a performance on the KNL 7250 of 960 Gflops/s meaning a 16\% of the peak performance. The multi node performances are discussed in section 2.3.1.

Wilson and Domainwall Kernels on Oakforest-PACS: I. Kanamori and H. Matsufuru developed for Bridge ++ two different implementations of a Wilson/Domainwall kernel [13]. Their strategy was to have a direct comparison of two radically different implementations: a simple one (impl-1) and a more aggressive one (impl-2). The most notable features of the two implementations are summarised in table 2 . 
Table 2. Features of the two kernels implementation.

\begin{tabular}{l|l}
\hline Simple (impl-1) see figure 8.a & Aggressive (impl-2) Grid-like see figure 8.b \\
\hline Simd vector is continuously packed in x-direction. & Simd vector is distributed to subdomains \\
No MPI parallelization in x- direction. & Non-blocking communications. \\
Blocking communications. & (partial) Loop tiling. \\
No manual prefetch. & Manual prefetch. \\
AVX512 intrinsics for arithmetics. & \\
\hline
\end{tabular}

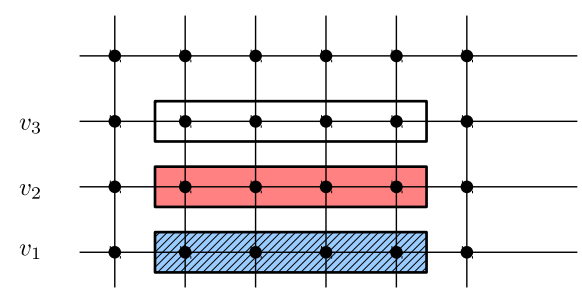

(a) Implementation 1 (Simple)

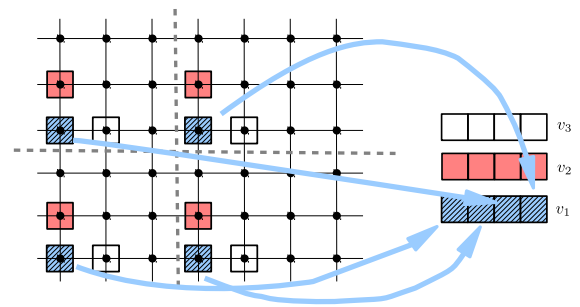

(b) Implementation 2 (Aggressive)

Figure 8. Data layout for two implementations of the Wilson/Domainwall operator.

The authors reported both on the single node and on the scaling performances of their codes on the OakForest-PACS PRIMERGY CX1640 M1 by Fujitsu (Intel Xeon Phi 7250 68C 1.4GHz (KNL) + Intel Omni-Path network topology: Full-bisection Fat Tree).

For the single node performances of the Wilson Dirac operators on a $32^{3} \times 64$ lattice results are:

- Impl-1: 241 GFlops (single precision, 4 threads/core: totally 256 threads), 147 GFlops (double, 4 threads/core)

- Impl-2: 339 GFlops (single, 2 threads/core), 174 GFlops (double, 2 threads/core)

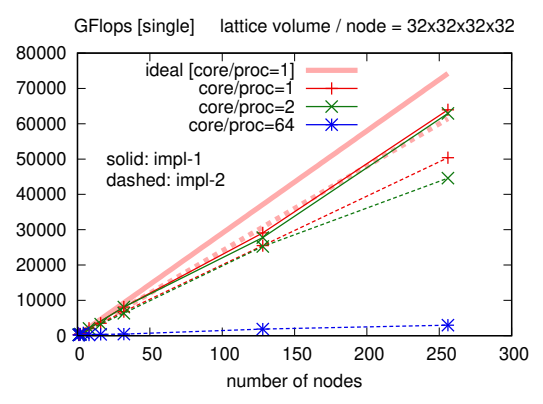

(a) Weak scaling

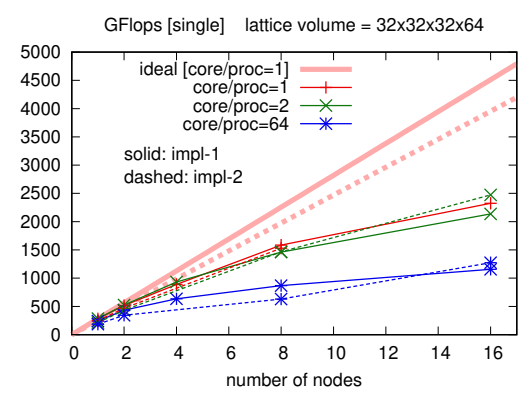

(b) Strong scaling

Figure 9. Scaling plots of the Wilson operator for impl-1 (solid) and impl-2(dashed). Ideal scalings are plotted with pink thick lines. 
They noted a strong dependence on the number of the threads/core for impl-1, with an increase of performance when using larger number of threads/core, while such behaviour is only marginally present in impl-2.

About the scaling performances the authors presented their studies for weak and strong scaling, as reported in figure 9.

They also reported on the single node and scaling performances of the domain wall operator with the impl-2 largely outperforming impl-1. The best performance for the single node study were obtained with 32 MPI processes and 4 threads/core: 395 GFlops for the single precision case and 197 GFlops for the double precision.

As a general tendency they noted that both for the Wilson and for the domainwall operators, the 1 and 2 cores/process cases give better performance than the 64 cores/process case.

MILC code performance on high end CPU and GPU supercomputer clusters: R. Li reported on the performances of three of the major QCD routines of the MILC code: the staggered multi-shift CG, Symanzik one-loop gauge-force, and the HISQ fermion force on the KNL architecture[14, 15]. For the staggered multishift CG and the Symanzik force the authors compared the performances obtained with the use of two different set of libraries: QOPQDP, the MILC baseline and QPhiX, the KNL optimised library developed in collaboration with Intel. The QPhiX library features the use a structure-of-array (SOA) data structure for improved cache reuse and a data layout similar to the Grid implementation.

The authors report an increase in perfomance of a factor 1.5 on the CG in using QPhiX on one KNL, while for the multinode application no substantial increase was found signalling that the algorithm was network bound on their test machine.

They also report on the weak scaling performances of the gauge and fermion forces with a scaling efficiency as high as $80 \%$ (see table 3 and figure 10).

Table 3. NERSC - Cori II single node and scaling performances: comparison among the MILC baseline and the QPhiX libraries.

\begin{tabular}{c|cc|cc}
\hline & \multicolumn{2}{|c|}{ multi-mass CG } & \multicolumn{2}{c}{ Symanzik Gauge force } \\
\hline & MILC b. & QPhiX & MILC b. & QPhiX \\
Gflops, single node & 70 & 120 & 60 & 380 (kernel) \\
Scaling factor $(16 \leq L \leq 32)$ & $0.3 \sim 0.55$ & $0.25 \sim 0.45$ & $0.4 \sim 0.85$ & $0.65 \sim 0.9$ \\
\hline
\end{tabular}

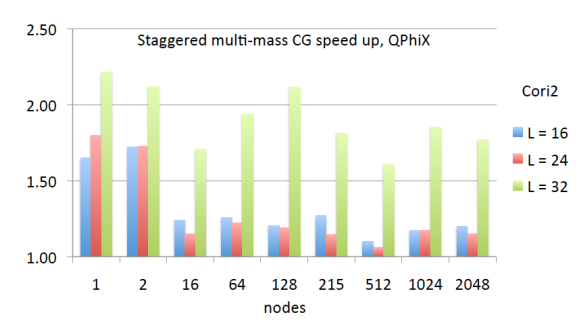

(a) CG speedup.

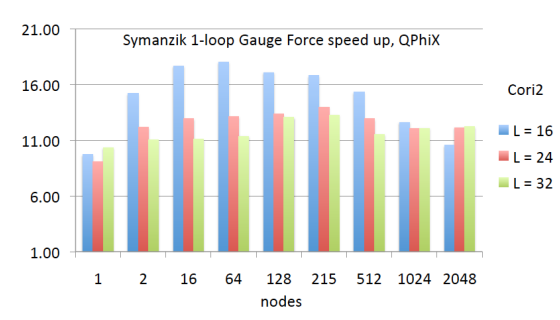

(b) Symanzik gauge speedup.

Figure 10. QPhiX library speedup over the MILC baseline on NERSC - Cori II 


\subsection{Tesla Volta}

Tesla V100 is latest NVIDIA accelerator based on the new NVIDIA Volta GV100 GPU [16]. The GV100 GPU includes 21.1 billion transistors with a size of $815 \mathrm{~mm} 2$. In figure 11.a there is a schematic breakdown of the GV100 Chip. A full GV100 GPU consists of six Graphics Processing Clusters (GPCs), 84 Volta Streaming Multiprocessors (SMs), 42 Texture Processing Clusters (TPCs) each including two SMs, and eight 512-bit memory controllers (4096 bits total).

The Tesla Volta features the second generation NVLink, that allows for both GPU-GPU and GPUCPU interconnections. In particular the GV100 supports up to 6 NVLink links with $25 \mathrm{~GB} / \mathrm{s}$ bandwith for a total aggregate bandwidth of $300 \mathrm{~GB} / \mathrm{s}$. In figure 11.b is reported a direct comparison of the hardware features with the previous GPU generation (Pascal).

To summarise the main improvements for lattice QCD come from:

- $1.5 x$ faster sustained memory bandwidth.

Volta sustains $95 \%$ of peak memory bandwidth in STREAM.

- Faster NVLink for better multi-GPU performance (300GB/S).

- Improved L1 Data Cache and Shared Memory subsystem.

The combined capacity is $128 \mathrm{~KB} / \mathrm{SM}$, more than 7 times larger than the previous generation (GP100) data cache.

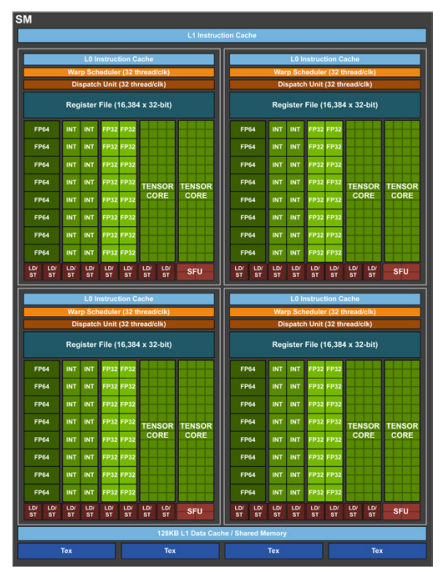

(a) GV100 structure.

\begin{tabular}{|c|c|c|c|}
\hline & P100 & V100 & Ratio \\
\hline Training acceleration & 10 TFLOPS & 120 TFLOPS & $12 x$ \\
\hline Inference acceleration & 21 TFLOPS & 120 TFLOPS & $6 \mathrm{x}$ \\
\hline FP64/FP32 & $5 / 10 \mathrm{TFLOPS}$ & $7.5 / 15$ TFLOPS & $\mathbf{1 . 5 x}$ \\
\hline HBM2 Bandwidth & $720 \mathrm{~GB} / \mathrm{s}$ & $900 \mathrm{~GB} / \mathrm{s}$ & $\mathbf{1 . 2 x}$ \\
\hline NVLink Bandwidth & $160 \mathrm{~GB} / \mathrm{s}$ & $300 \mathrm{~GB} / \mathrm{s}$ & $\mathbf{1 . 9 x}$ \\
\hline L2 Cache & $4 \mathrm{MB}$ & $6 \mathrm{MB}$ & $\mathbf{1 . 5 x}$ \\
\hline L1 Caches & $1.3 \mathrm{MB}$ & $10 \mathrm{MB}$ & $\mathbf{7 . 7 x}$ \\
\hline
\end{tabular}

(b) Volta vs. Pascal.

Figure 11. NVIDIA Volta technical details.

\subsubsection{Contributions}

Developing QCD Algorithms For NVIDIA GPUs Using the QUDA Framework: QUDA is an NVIDIA GPU library for QCD simulations, it can be used both to accelerate traditional lattice QCD applications or as standalone framework. It supports many different fermion discretizations, and features many algorithms like Adaptive Multigrid, deflation and Block Krylov space methods, using various techniques such as mixed-precision and communication hiding to maximise performance [17-19]. This library has been around for roughly ten years and has kept on showing increased performances as 
the hardware evolved with time, showing very good properties of portability across the NVIDIA GPU generations (see figure 12.a). In particular for the application of the same Hisq Dslash over multiple righthand sides the scale factor from Pascal to Volta is roughly a factor two (see figure 12.b).

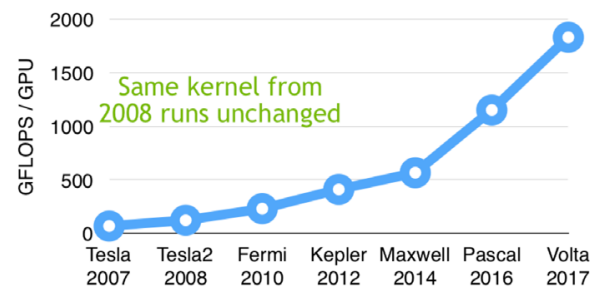

(a) Single precision Wilson-Dslash operator performances for different hardware

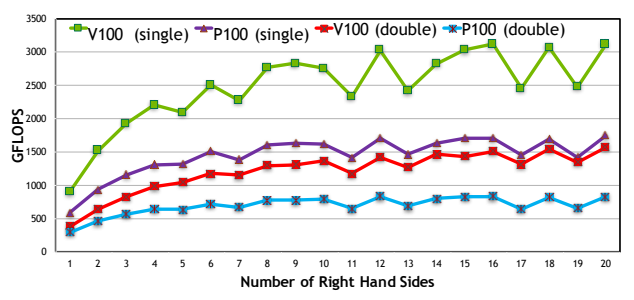

(b) Hisq Dslash for multiple rhs

Figure 12. QUDA single GPU performances

K. Clark and M. Wagner [20, 21] presented the scaling performances of the QUDA libraries both for weak and strong scaling on a cluster equipped with 8xP100 (Pascal) GPUs nodes, with NVLINK as intra-node GPU connection and using 4x EDR for inter-node communication (see figure 13.a).

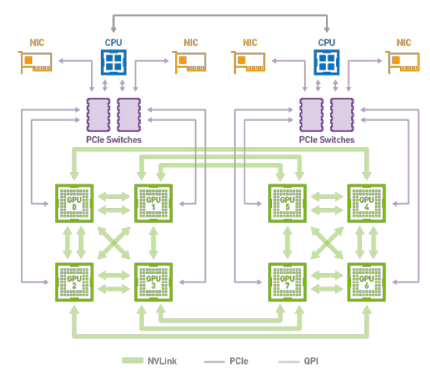

(a) Node structure.

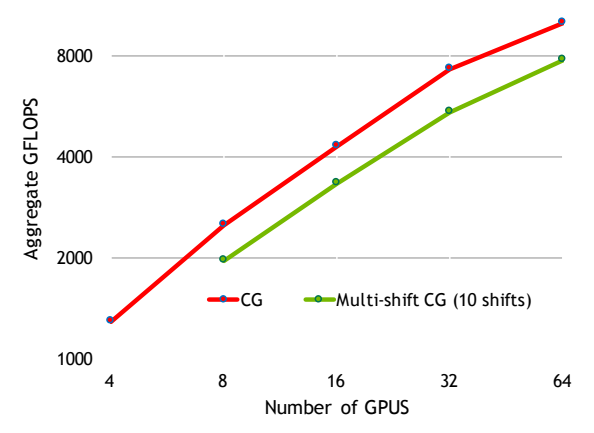

(c) Clover strong scaling.

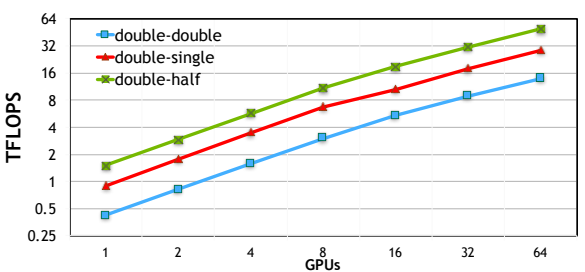

(b) Solver (mixed precision Shamir) weak scaling.

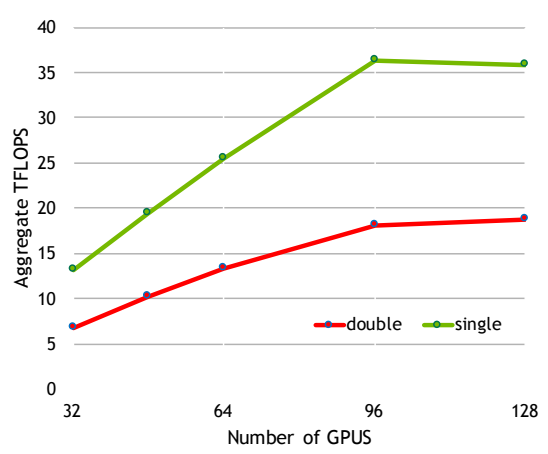

(d) HISQ strong scaling.

Figure 13. QUDA multi-GPU scaling performances. 
The code shows good scaling properties, but what is particularly instructive is the breakdown of the steps needed to achieve such scaling behaviour:

- For the intra-node, direct peer-to-peer communication.

- Direct access to the network interface through GPU Direct for inter-node communication.

- Topology aware communications, in order to avoid multiple hops among the PCIe switches.

\section{Performance Portability Strategies for Grid C++ Expression Templates:}

M. Lin presented successes and challenges encountered in porting the Grid $\mathrm{C}++$ expression template to GPU-based systems and exploring extensively different approaches to integrate CUDA, OpenACC and Just-In-Time compilation [22].

In order to port the Grid library in an "effortless" way the authors strategy was to map the Grid's block into a vector and the threads into the elements of a vector. This approach greatly simplifies code integration, because most of the underlying code structures are shared.

They report on the performance studies in the case of an $\mathrm{SU}(3) \times \mathrm{SU}(3)$ streaming test, showing that their approach is capable of achieving good performance (see figure 14).

In particular in figure 14.b the authors show that the performance reaches its maximum with a block size of 16 threads, or equivalently a vector length of 16 single-precision complex numbers.

They also comment on possible improvements to avoid the need of large vector length.

The porting of the Grid Dslash operator is under active development.

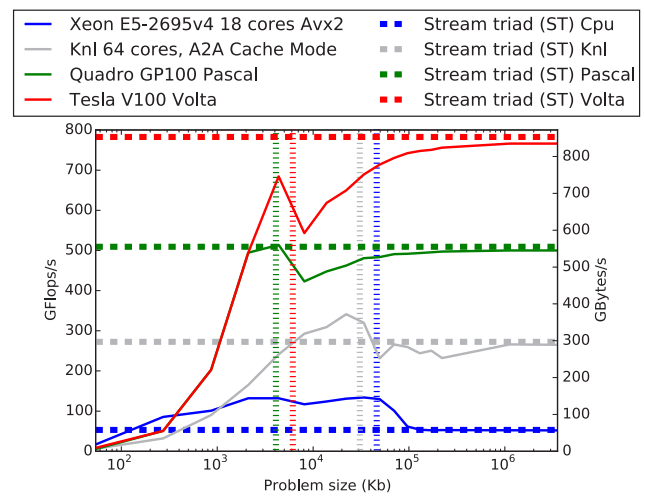

(a) Performance over several architectures. The vertical lines indicate the cache size, the horizontal ones show the memory bandwidth obtained in a stream triad test.

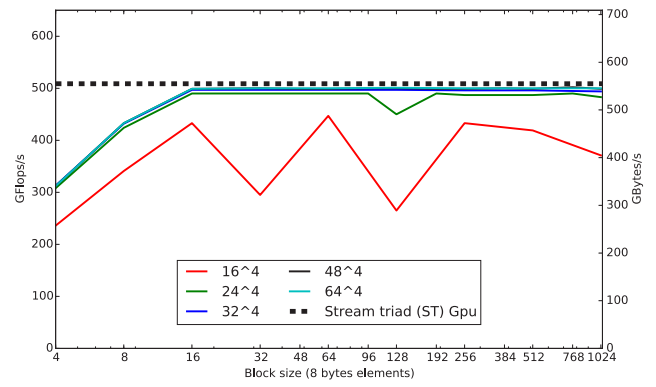

(b) Performance as a function of the vector length in a Quadro GP100.

Figure 14. $\mathrm{SU}(3) \times \mathrm{SU}(3)$ streaming test over multiple architectures.

\subsection{Intel Omni-Path}

Omni-Path (OPA) is the new high-performance communication architecture designed by Intel. It is designed specifically for HPC purpose with low communication latency, low power consumption and a high throughput. It is developed by Intel as the on-ramp to exascale computing.

The OPA is designed and optimised for the MPI communication model and it is capable of a scalable All-To-All communication. 
One of the principal differences with other comparable products is that all the network functions are executed by the hosting system CPU as the OPA doesn't equip any co-processor. As such overlapping communications and computations will anyway have to rely on a common set of resources.

One of the key issues that was highlighted multiple times during the conference was the unavailability of the threaded version of the communication driver in the Omni-Path software stack. This limitation has been overcome in the recent major upgrade [23].

In our community already quite a few production machines are equipped with this new fast network and reports on their efficiency and scalability were presented during the conference.

\subsubsection{Contributions}

An in-depth evaluation of the Intel Omni-Path network for LQCD applications and An implementation of the DD- $\alpha$ AMG multigrid solver on Intel Knights Landing: P. Georg and D. Richtmann presented the porting of their collaboration codebase onto the new QPACE3 machine, based on KNL nodes [24]. More specifically they presented their efforts in porting of the DD- $\alpha$ AMG solver. QPACE 3 is equipped with the new OPA interconnection and a very in-depth analysis of the performances and issues associated with this new hardware was presented. The authors have highlighted as key performance factors the network latency and the message rate. They noted that, when running on a single or a small number of nodes, the standard parallelization approach with one MPI rank per node and one or more threads on each core gives the best performances on their code. On the contrary, when running on larger number of nodes, they found beneficial using multiple MPI ranks per processor.

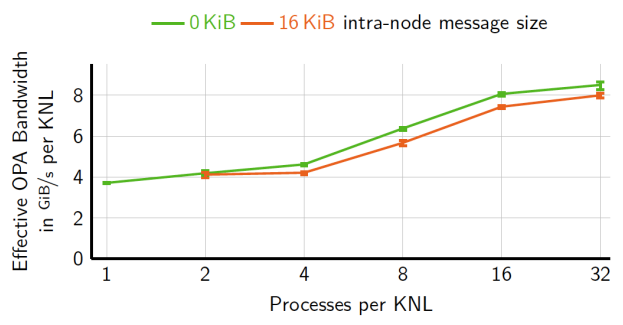

(a) Bandwith as function of number of processes

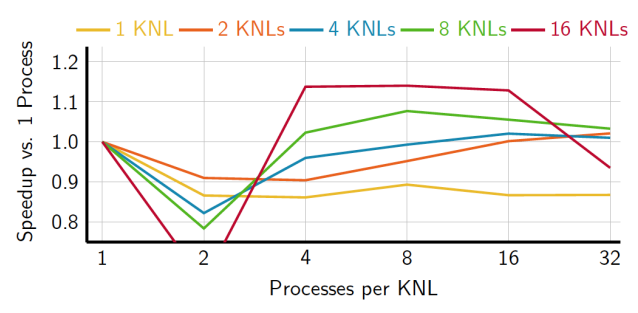

(b) Speedup as function of the number of processes

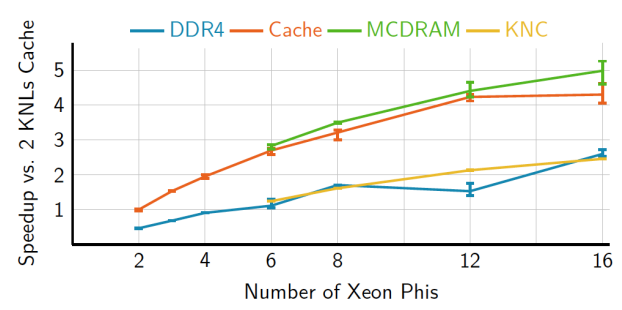

(c) DD- $\alpha$ AMG strong scaling on KNL and KNC

Figure 15. Performance analysis of the Intel OPA network.

In particular in figure 15.a they presented the halo-exchange performances on $16 \mathrm{KNL}$. They present the aggregate bandwidth in $\mathrm{GiB} / \mathrm{s}$ of a single $\mathrm{KNL}$ as function of the number of processes for 
inter-node message size of $512 \mathrm{KiB} /$ (number of processes per node) and 0 and $16 \mathrm{KiB}$ intra-node message size.

In figure 15.b it is reported the speedup factor of the DD- $\alpha \mathrm{AMG}$ for various size of cluster of KNLs vs. number of processes per KNL.

Finally in figure 15.c they present the comparison in speedup between KNC and KNL for the off-chip strong scaling of DD- $\alpha$ AMG.

Grid software status and performance: The Grid developers have analysed the performances of the OPA infrastructure and compared them with the performances of other network hardware. They have studied the Grid's halo exchange performances on three different machines respectively equipped with Intel-OPA, Mellanox-EDR and Cray-Aries. They highlighted that a single MPI-process per node on OPA is not capable of saturating the bandwith for the typical message size used by Grid, leading to the need of subdividing the problem within a node, hence increasing the unwanted intranode communication. A short summary of their findings is reported on table 4.

Table 4. Grid halo-exchange best performances on multiple architecture

\begin{tabular}{llllll}
\hline Machine & Node type & Network & Bidi peak GB/s & 1 rank per node GB/s & 4 ranks per node GB/s \\
\hline BNL & KNL & Dual OPA & 50 & 14 & 44 \\
Cori2 & KNL & Aries & $\sim 16$ & 11 & 11 \\
SGI & ICE-X Xeon & Dual EPR & 50 & 44 & 44 \\
\hline
\end{tabular}

In figure 16 it is reported a more direct comparison between Intel OPA and Mellanox EDR dual rail on SGI ICE-X Xeon E5-2690 2.4Ghz nodes(only best performances).

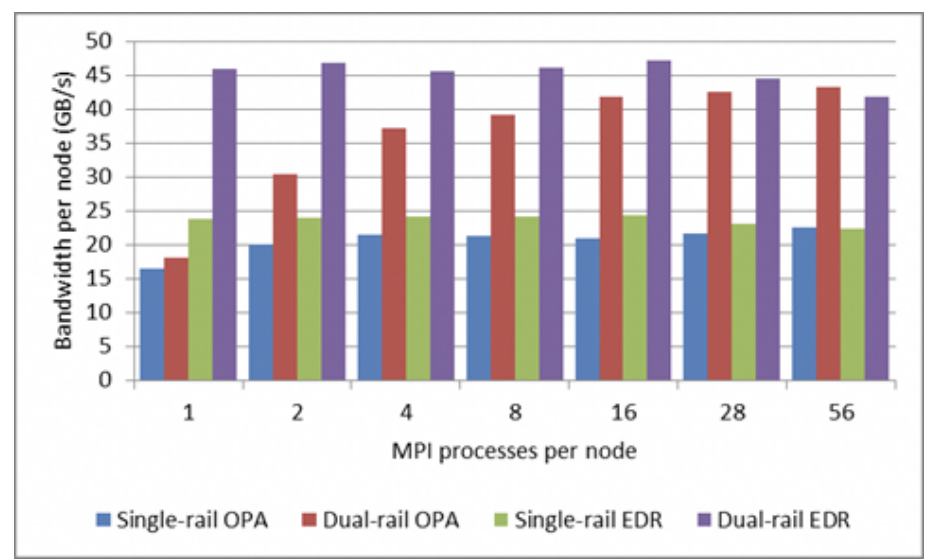

Figure 16. Comparison of performances of the Grid's halo exchange between Intel OPA and Mellanox EDR dual rail (courtesy: SGI HPE)

The authors have identified a possible workaround specific for Omni-Path: they have used MPI3 shared memory extension to accelerate intranode communications. The MPI-3 shared memory features enable programmers to create regions of shared memory that are accessible by the MPI processes. Hence the Grid developers have used multiple MPI communications per node and ensured that different ranks on the same node assume cartesian coordinates in cube of next-neighbours. They 
differentiate between interior and external communication and use for the former a direct copy into shared memory. A breakdown of the computation and communications timings is reported in figure 17.b. However communication overhead still dominates on small local volumes.

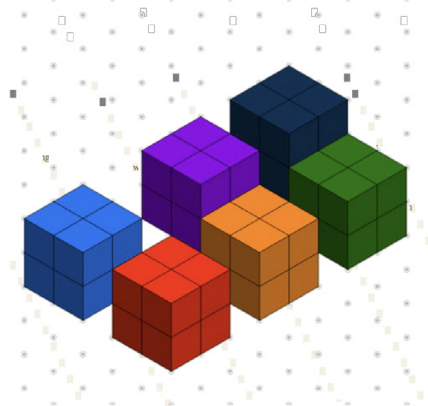

(a) Geometrical mapping: different ranks on the same nodes belong to a cube of next-neighbours.

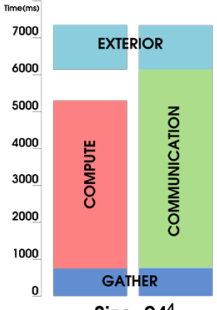

Size: $24^{4}$
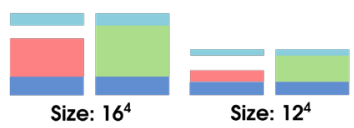

(b) Timing breakdown of the application of the Dirac operator for different lattice sizes.

Figure 17. Grid MPI-3 shared memory communicators.

It must be noted that at present the Grid suite fully supports threaded MPI communications, taking advantage of the latest driver release, an evolution that has drastically improved the communication performances on the OPA hardware, compared to what was reported during the conference[25].

Half Precision Communications A more generic workaround takes advantage of the tolerance that some inverter algorithms show against the damaging of the neighbour information. C. Kelly and P. Boyle showed that for a given inner conjugate gradient tolerance, matrix inverter precision can be kept roughly constant even if the neighbour information is transmitted only in half-precision. The author have found a $45 \%$ reduction in communication time swapping to half precision communication. They

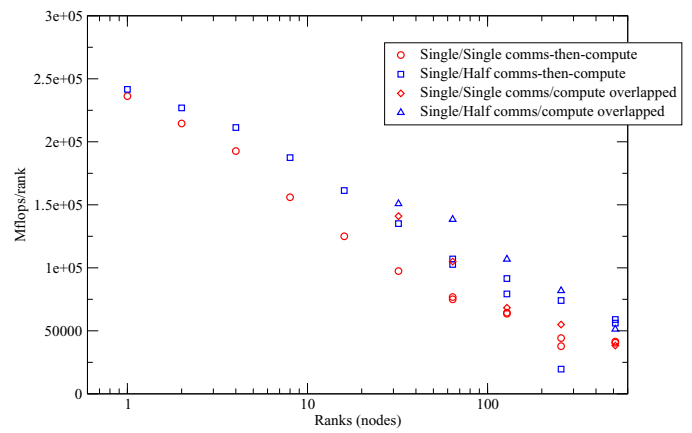

Figure 18. Strong-scaling performance of Grid halo exchange for single and half precision communication on Cori II. 
also note that a careful implementation of the compress/decompress procedure was needed not to add any additional overhead. The strong scaling investigation of this algorithm for the halo-exchange of Grid is reported in figure 18, the labels single/single and single/half refer to the overall floating point precision of the Dslash and the precision of the communications.

\section{Conclusions}

Data Motion, Memory and Cache bounds are defining the performances of QCD codes. Being capable to face them all at the same time is the challenge that every lattice QCD programmer is facing nowadays. We are moving towards a more hybrid kind of programming although there is always the hope that CPU evolution will move along compiler's capability of optimising code, e.g taking advantage in auto-vectorising large vector units (AVX512). GPU and in particular the QUDA libraries implementation have shown very good performances, proving that the coding model of GPU is portable over the hardware evolution with little coding cost. Concerning the new network hardware by Intel, all concerns seem to be software related and might have already been solved in the new version of the PSM2 driver. Quite likely we will discover the new performances in next year's report.

Acknowledgements: I would like to thank the conference's organizers for the invitation to present this review. I am indebted to Guido Cossu for insightful comments at all stages of this work. Acknowledgements for support go to the STFC Consolidated Grants ST/L000350/1 and ST/P000479/1. 


\section{References}

[1] G.E. Moore, Electronics 38 (1965)

[2] TOP500, https://www.top500.org

[3] P.A. Boyle, PoS LATTICE2016, 013 (2017)

[4] J. Jeffers, J. Reinders, A. Sodani, Intel Xeon Phi Processor High Performance Programming: Knights Landing Edition (Elsevier Science, 2016), ISBN 9780128091951, https://books . google.ch/books?id=DDpUCwAAQBAJ

[5] C. Research, Performance optimization for intel xeon phi x200 product family (2016), https: //colfaxresearch.com/how-knl/

[6] K. Raman, Optimizing memory bandwidth in knights landing on stream triad (2016), https://software.intel.com/en-us/articles/ optimizing-memory-bandwidth-in-knights-landing-on-stream-triad

[7] S. Durr, Optimization of the Brillouin operator on the KNL architecture, in 35th International Symposium on Lattice Field Theory (Lattice 2017) Granada, Spain, June 1824, 2017 (2017), 1709.01828, http://inspirehep.net/record/1621813/files/arXiv: 1709.01828.pdf

[8] S. Durr, G. Koutsou, PoS LATTICE2016, 249 (2016), 1610. 06798

[9] S. Durr, G. Koutsou (2017), 1701.00726

[10] P. Boyle, A. Yamaguchi, G. Cossu, A. Portelli (2015), 1512.03487

[11] P.A. Boyle, G. Cossu, A. Yamaguchi, A. Portelli, Data parallel c++ mathematical object library (2016), https://github.com/paboyle/Grid

[12] P.A. Boyle, G. Cossu, A. Yamaguchi, A. Portelli, PoS LATTICE2015, 023 (2016)

[13] I. Kanamori, H. Matsufuru, Wilson and Domainwall Kernels on Oakforest-PACS, in 35th International Symposium on Lattice Field Theory (Lattice 2017) Granada, Spain, June 1824, 2017 (2017), 1710.07226, http://inspirehep.net/record/1631665/files/arXiv: 1710.07226.pdf

[14] C. DeTar, S. Gottlieb, R. Li, D. Toussaint, MILC Code Performance on High End CPU and GPU Supercomputer Clusters, in 35th International Symposium on Lattice Field Theory (Lattice 2017) Granada, Spain, June 18-24, 2017 (2017)

[15] MILC collaboration, Milc collaboration code for lattice qcd calculations, https://github. $\mathrm{com} / \mathrm{milc}-\mathrm{qcd} / \mathrm{milc}$-qcd

[16] L. Durant, O. Giroux, M. Harris, N. Stam, Inside volta: The world's most advanced data center gри (2017), https://devblogs.nvidia.com/parallelforall/inside-volta/

[17] M.A. Clark, R. Babich, K. Barros, R.C. Brower, C. Rebbi, Comput. Phys. Commun. 181, 1517 (2010), 0911. 3191

[18] R. Babich, M.A. Clark, B. Joo, G. Shi, R.C. Brower, S. Gottlieb, Scaling Lattice QCD beyond 100 GPUs, in SC11 International Conference for High Performance Computing, Networking, Storage and Analysis Seattle, Washington, November 12-18, 2011 (2011), 1109.2935, http: //inspirehep.net/record/927455/files/arXiv:1109.2935.pdf

[19] R. Babich, K. Barros, R. Brower, M. Clark, J. Foley, J. Giedt, S. Gottlieb, B. Joó, C. Rebbi, G. Shi et al., Quda: A library for qcd on gpus, https://lattice.gi thub.io/quda/

[20] K. Clark, M. Wagner, Developing QCD Algorithms For NVIDIA GPUs Using the QUDA Framework, in 35th International Symposium on Lattice Field Theory (Lattice 2017) Granada, Spain, June 18-24, 2017 (2017) 
[21] K. Clark, M. Wagner, Approaching QUDA 1.0, in 35th International Symposium on Lattice Field Theory (Lattice 2017) Granada, Spain, June 18-24, 2017 (2017)

[22] P.A. Boyle, M.A. Clark, C. DeTar, M. Lin, V. Rana, A.V. Avilés-Casco, Performance Portability Strategies for Grid C++ Expression Templates, in 35th International Symposium on Lattice Field Theory (Lattice 2017) Granada, Spain, June 18-24, 2017 (2017), 1710.09409, http://inspirehep.net/record/1632761/files/arXiv: 1710.09409.pdf

[23] Intel, Psm2 library source code, https://github.com/01org/opa-psm2/releases

[24] P. Georg, D. Richtmann, T. Wettig, DD- $\alpha A M G$ on QPACE 3, in 35th International Symposium on Lattice Field Theory (Lattice 2017) Granada, Spain, June 18-24, 2017 (2017), 1710.07041, http://inspirehep.net/record/1631654/files/arXiv: 1710.07041.pdf

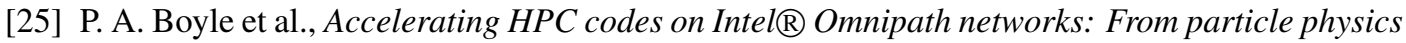
to Machine Learning, in to appear (2017) 